\title{
Health Tips
}

\author{
Vaishnavi Satheesh* \\ Dietitian, Content Developer, India \\ *Corresponding Author: Vaishnavi Satheesh, Dietitian, Content Developer, India.
}

Received: August 07, 2019; Published: September 13, 2019

Confused about your diets? It is now time to consult a qualified dietitian or nutritionist. The idea of following a fad diets which is planned by fake people and published through Mr. Google, has nothing to change your body type. Every diet should be tailor made, not a same diet suits all. I place it as important health tip because, in recent times I can meet some people who simple follows diet and medication by surfing the internet. They don't understand the real consequences that they are going to face in few years later in their life. Having a real concern over your health, I post this as first and foremost health tip, that, never ever follow a self designed diet or medication unless you are specialized in it.

Honey that is included in your early morning drink aids in multi tasks inside our body. As I usually states honey is a magic sweetener, which imparts beneficiaries in any ways it is been taken. Surprised? I will get you clarity. Honey has anti inflammatory property that provides you from being building up your immunity especially in kids. A kid who strives hard to put on weight, addition of honey to a glass of warm milk increase the weight along with protecting the kid from catching frequent cold. As milk is one among the super nutritious food, the honey adds to its nutritional value. Some people have the habit of having Ginger lemon water mixed with honey in empty stomach. Though they might add honey for sweetness, the honey being the secret ingredient in helping the person loses his toxins from the body. Worried about your kid catching cold and dry cough? Here is the best remedy that add a spoon of honey to a warm water and make your kid consume it regularly, along with boosting their immunity, the cold and dry cough may run away as the honey have the soothing effects.
This tip for our little prince and princess in our home. To make them even sharper and observer, I recommend 2 walnuts a day. Make your kid a habit of consuming 2 walnuts along with their healthy breakfast to improve their mind alertness and thus improving their academic skills. Walnuts can be included in any forms like mixed with juices, infused in batter or dough, or a simple walnut as it is. Holding the shape of the brain, it insists us the beneficiaries it provides to the brain when we consume it on regular basis. Have a try and update me!

The spices we use in our regular cuisine have some nutritional specialties. In that way, ginger is one of the most exciting spices that help in the more important functions of our body. The powerful antioxidant, gingerol possess many medicinal qualities thus making it as a best option to cure nausea, vomiting and morning sickness. This ginger is the best ingredient to use it in regular regimen, as it has many anti-inflammatory properties, which aids in preventing many illnesses. As the best remedy for chronic indigestion, ginger holds a personal space in many of the kitchen.

Weight loss plateau, is the most fearful phrase in the journey of a person with weight loss. After successfully shedding extra kilos, there comes a phase called weight loss plateau, where there is no weight loss or inch loss. This is very much common, and can be eradicated with high amount of exercise and strict dieting. Hence, when on weight loss plateau, feel free to get in touch with your health expert and make ways to get through the plateau. Don't lose hope and stops dieting, because, when you skip your diet, your condition may reverse at times. 
Are you on your weight loss journey? Confused about eating options while a day out? Don't worry, try out some paneer recipes, baked or grilled chickens, or vegetable filled rolls, to fill your tummy with, and the remaining parts be filled with your soups and low calorie desserts like fresh juices and lemonades.

Suffering from mental sickness? Then it is now the time to enjoy a chocolate. Yes! Consuming a dark chocolate may reduce the mental sickness and thus boosting the brain energy. It produces calm and relaxed mood. When stressed under heavy work pressure, make sure you have you chocolate soon, to feel energized. No chocolates, try a cocoa based product!

Menopause, a major milestone in a woman's life, is characterized by mood swings and hormonal fluctuations. Thus proper nutrition is a key element, for crossing this phase effectively. Fruits like banana, mango, eggs, milk and sesame are some of the super foods that aids in treating the sleep troubles and mood swings. Fruits are the best partners at this phase, as it contains fiber and potassium, these aids in preventing the fluid retention.

Though there is no specific timing for foods, the best time is there, that makes the foods richer in expressing their quality. In that way, fruits should be taken as the first thing in the empty stomach of every morning, or as a stand-alone food in between meals. Some people have the habit of inclusion of these fruits either before or after meals. This should be avoided. Fructose in the fruits makes the digestion of fruits to alter resulting in burning sensation when taken with foods.

Is your child over reacting to certain situations? Then have a check on their sleeping pattern and timings. Irregular sleeping patterns or improper timings should face the situations with less confidence, making them arrogant and adamant in certain situations. Actually the moms are so much with embarrassment, with the kid's activities. The true fact is that we should make the sleeping pattern correct. Make your child or little toddler to enjoy the sleep.
Overnight oats is the simplest recipe that aids in quick weight loss. Oats are healthier, and that it provides all the essential nutrients in simpler forms. This oat meal is good source of iron, thus preventing from anemia, which is a gate pass to many of the disorders.

\section{Volume 3 Issue 10 October 2019} (c) All rights are reserved by Vaishnavi Satheesh. 